2162-8769/2016/5(3)/P1/7/\$33.00 @ The Electrochemical Society

\title{
Thermal Shields for Heat Loss Reduction in Siemens-Type CVD Reactors
}

\author{
A. Ramos, J. J. Valdehita, J. C. Zamorano, and C. del Cañizo \\ Instituto de Energía Solar - Universidad Politécnica de Madrid, ETSI Telecomunicación, 28040 Madrid, Spain
}

\begin{abstract}
The use of thermal shields to reduce radiation heat loss in Siemens-type CVD reactors is analyzed, both theoretically and experimentally. The potential savings from the use of the thermal shields is first explored using a radiation heat model that takes emissivity variations with wavelength into account, which is important for materials that do not behave as gray bodies. The theoretical calculations confirm that materials with lower surface emissivity lead to higher radiation savings. Assuming that radiation heat loss is responsible for around $50 \%$ of the total power consumption, a reduction of $32.9 \%$ and $15.5 \%$ is obtained if thermal shields with constant emissivities of 0.3 and 0.7 are considered, respectively. Experiments considering different thermal shields are conducted in a laboratory CVD reactor, confirming that the real materials do not behave as gray bodies, and proving that significant energy savings in the polysilicon deposition process are obtained. Using silicon as a thermal shield leads to energy savings of between 26.5-28.5\%. For wavelength-dependent emissivities, the model shows that there are significant differences in radiation heat loss, of around $25 \%$, when compared to that of constant emissivity. The results of the model highlight the importance of having reliable data on the emissivities within the relevant range of wavelengths, and at deposition temperatures, which remains a pending issue. (C) 2016 The Electrochemical Society. [DOI: 10.1149/2.0171603jss] All rights reserved.
\end{abstract}

Manuscript submitted November 18, 2015; revised manuscript received December 18, 2015. Published 00 0, 2016.

\section{Scope}

$90 \%$ of the polysilicon currently produced worldwide is demanded by the photovoltaic (PV) market, leaving the remaining amount for 3 the microelectronics industry! ${ }^{[12}$ The chemical route -via chemical 24 vapor deposition (CVD) of high purity trichlorosilane (TCS) on a hot filament, the so-called Siemens technology- currently dominates polysilicon production. High quality polysilicon is obtained, at the expense of high energy consumption $\frac{314}{36}$

In the case of polysilicon for PV (also known as solar grade silicon) the process accounts for between a quarter and a third of the total energy consumption $[5+7$ Thus, lowering the energy consumption of the Siemens process is essential to achieving the two wider objectives for silicon-based PV technology: low production cost and low energy payback time.

Furthermore, current price levels also press polysilicon producers to reduce their production costs even more if they are seeking a sustainable business $[8$

As radiation heat loss is the major contributor to energy consumption,, 9 in this work the potential of thermal shields to reduce radiation heat loss in an industrial Siemens reactor is studied. Thermal (radiation) shields have been implemented in a number of CVD reactors; e.g. for layer deposition in superconducting devices or for the epitaxial growth of silicon layers 10 [1] Several proposals have recently been made for polysilicon production,,$\frac{12}{14}$ but to our knowledge quantitative analysis supported by experimental data has not been provided in any of them.

Radiation heat loss as regards thermal shields in a Siemens-type CVD reactor is studied first here using a theoretical model. Then, the theoretical results are compared with the experimental results in a laboratory Siemens reactor. Discussion of the latter will offer insights into the accuracy of theoretical calculations depending on the thermal shield materials' optical properties, highlighting the relevance of the variation in optical properties with the wavelength for thermal shield materials.

Radiative heat transfer.-Radiative heat transfer - also known as 60 thermal radiation - describes the science of heat transfer caused by

${ }^{\text {z} E-m a i l: ~ a l b a . r a m o s @ i e s-d e f . u p m . e s ~}$

electromagnetic waves. These electromagnetic waves have the property of traveling through a vacuum or matter-containing media. The temperature of the radiant body governs the thermal radiation emission, and it occurs in the 0.1 to $100 \mu \mathrm{m}$ wavelength range.!15]16 It is not the aim of this section to explain the thermal radiation phenomenon in detail, but to describe a number of concepts and properties of the radiation heat transfer mechanism that will support the arguments we develop in this document.

As regards the radiation properties, four dimensionless magnitudes are defined: absorptance $(\alpha)$, reflectance $(\rho)$, transmittance $(\tau)$ and emissivity $(\varepsilon)$. Absorptance, reflectance and transmittance are defined as the ratio of the total amount of radiation absorbed, reflected or transmitted by a surface to the total amount of radiation incident on the surface, respectively. The emissivity Emissivity is defined as the ratio of the power per unit area radiated by a surface to the power per unit area radiated by a black body at the same temperature. These properties for real surfaces are dependent on temperature, direction and wavelength. The relationship indicated in Equation 1 is obtained by applying the energy balance to any real surface.

$$
\alpha+\rho+\tau=1
$$

In addition, according to Kirchhoff's law, all opaque surfaces ( $\tau=$ 0) reach $\varepsilon_{\lambda}(\lambda, T)=\alpha_{\lambda}(\lambda, T)$.15]16

A black body is defined as any body that emits and absorbs the maximum possible radiation in all wavelengths, that is: $\alpha=1, \rho=$ $\tau=0$. Plack's law defines the spectral radiated power of a black body. In addition, according to Stefan-Boltzmann's law the expression for the total radiation emitted per unit area of a black body is indicated in Equation 2; where $T$ is the temperature and $\sigma$ the Boltzmann constant.

$$
E_{b}(T)=\sigma T^{4}
$$

However, the majority of the surfaces do not behave as black bodies; thus, the gray body concept arises. A gray body is any opaque body ( $\tau=0, \alpha+\rho=1$ ) whose reflectance, absorptance and emissivity properties are non dependent on the wavelength. The behavior of many real surfaces can be approximated to that of a gray body; in Equation 3 the expression of the total radiation emitted per unit area of a gray body is presented.

$$
E_{g}(T)=\varepsilon_{g} \sigma T^{4}
$$

The parameter $\varepsilon_{g}$ corresponds to the emissivity of a gray body.

But, being more rigorous, real surfaces do not necessary behave as gray bodies, and their properties vary with the wavelength for a 
98 given temperature. These surfaces radiate a different fraction $\varepsilon_{\lambda}$ at 99 each wavelength; thus, the expression of the total radiation emitted 100 per unit area of a real surface is indicated in Equation 4. Note that the 101 parameter $\varepsilon_{r}$ in Equation 4 is calculated by means of Equation 5: that 102 is, integrating $\varepsilon_{\lambda}$ along all the radiation spectrum.

$$
E_{\text {real }}(T) \cong \varepsilon_{r} \sigma T^{4}
$$

103

$$
\varepsilon_{r}=\frac{\int_{0}^{\infty} \varepsilon_{\lambda} E_{b \lambda} d \lambda}{\int_{0}^{\infty} E_{b \lambda} d \lambda}
$$

104 105 106

Radiation heat loss model. - A radiation heat loss model for heat 117 loss calculations in a Siemens-type reactor was presented and de118 scribed in detail in Ref. 20, and validated in Ref. 21. It is further 119 developed within the framework of this research to broaden its appli120 cability and account for materials that do not behave as gray bodies.

121 One parameter needs to be defined for radiation heat loss calcu122 lations: radiosity $(J)$, the rate of outgoing radiant heat per unit area 123 from a surface. It is the sum of the directly emitted heat flux $(E)$ and 124 the reflected incoming radiant heat flux from the surface $(G)$. The 125 fraction of heat flux from one surface to another is determined by the 126 so-called configuration factor, or geometrical factor. The calculation 127 of the configuration factors $\left(F_{i-j}\right)$ is made using a geometric Hottel's 128 crossed-string method. ${ }^{22}$ In the present case note that the rods and the 129 reactor wall have a cylindrical geometry.

130 If the material properties, the geometrical arrangement, the surface 131 temperatures and the incoming and directly emitted radiant heat flux 132 are known, the net heat flux exchanged $(Q)$ in Watts from any surface $133\left(S_{i}\right)$, is obtained from the difference between the radiosity and the 134 incoming radiant heat flux. Then, the net radiation heat flux exchanged 135 for a certain surface $i$ can be expressed as shown in Equation 6 .

$$
Q_{i}=S_{i} \cdot\left(J_{i}-G_{i}\right)=S_{i} \cdot J_{i}-\sum_{j=1}^{n} S_{j} \cdot F_{j-i} \cdot J_{j}
$$

136 For a Siemens reactor of $n$ - 1 rods, a $n$-equations system needs to 137 be solved, as the reactor wall is considered as an additional surface. 138 The radiosities of each surface $(J i)$ are the unknowns of the system. 139 The temperature of the rod surfaces and the reactor wall is known, as 140 is the corresponding surface emissivities. Once the $J_{i}$ is obtained for 141 the $n$ surfaces, the incoming radiant heat flux per unit area $\left(G_{i}\right)$ is also 142 known. Thus, the net radiation heat exchanged by each surface $\left(Q_{i}\right)$ 143 is obtained by substituting $J_{i}$ and $G_{i}$ in Equation 6 .

144 To account for emissivity variations with the wavelength, radiation 145 heat loss is obtained by means of Equations 7, 8, 9 and 10, which are 146 solved independently for each wavelength

$$
S_{i} \cdot \frac{1}{1-\varepsilon_{i}(\lambda)} \cdot J_{i}(\lambda)-\sum_{j=1}^{n} S_{i} \cdot F_{i-j} \cdot J_{j}(\lambda)=S_{i} \cdot \frac{\varepsilon_{i}(\lambda)}{1-\varepsilon_{i}(\lambda)} \cdot \sigma \cdot T_{i}^{4}
$$

147

$$
E_{i}(\lambda)=\varepsilon_{i}(\lambda) \cdot \sigma \cdot T_{i}^{4}
$$

148

$$
G_{i}(\lambda)=\frac{1}{1-\varepsilon_{i}(\lambda)} \cdot\left(J_{i}(\lambda)-E_{i}(\lambda)\right)
$$

$$
Q_{i}(\lambda)=S_{i} \cdot\left(J_{i}(\lambda)-G_{i}(\lambda)\right)
$$

where $i=1, \ldots, n$.

The net heat flux exchanged $\left(Q_{i}\right)$ in Watts by any surface $\left(S_{i}\right)$, is obtained by integrating $Q_{i}(\lambda)$ along all the radiation spectrum. In Equation 11 the net heat flux exchanged by a surface is presented; $E_{b}(\lambda)$ is the total radiation emitted per unit area of a black body indicated in Equation 2.

$$
Q_{i}=\frac{\int_{0}^{\infty} Q_{i}(\lambda) E_{b}(\lambda) d \lambda}{\int_{0}^{\infty} E_{b}(\lambda) d \lambda}
$$

This radiative model allows extra surfaces in the Siemens reactor 156 to be considered and their positive or negative effect on heat savings $\quad 157$ studied. This can be the case of a thermal shield. A thermal shield 158 is a cylinder surrounding the polysilicon rods and placed between 159 them and the reactor wall. The presence of this shield may block 160 a significant part of the radiated heat that otherwise would be lost 161 through the reactor wall.

Now, the net heat flux exchanged $\left(Q_{i}\right)$ in Watts by any surface $\left(S_{i}\right)$, is again obtained by integrating $Q_{i}(\lambda)$ along all the radiation spectrum; but by replacing Equation 7 with Equations $12-15$ (where $i=1, \ldots, m-1$, and $m$ is the number of thermal shields considered).

$$
S_{i} \cdot \frac{1}{1-\varepsilon_{i}(\lambda)} \cdot J_{i}(\lambda)-\sum_{j=1}^{m} S_{i} \cdot F_{i-j} \cdot J_{j}(\lambda)=S_{i} \cdot \frac{\varepsilon_{i}(\lambda)}{1-\varepsilon_{i}(\lambda)} \cdot \sigma \cdot T_{i}^{4}
$$$$
S_{m} \cdot \frac{1}{1-\varepsilon_{m}(\lambda)} \cdot J_{m}(\lambda)-\sum_{j=1}^{m} S_{m} \cdot F_{m-j} \cdot J_{j}(\lambda)=S_{m} \cdot \frac{\varepsilon_{m}(\lambda)}{1-\varepsilon_{m}(\lambda)} \cdot \sigma \cdot T_{m}^{4}
$$

$$
\left(S_{m} \cdot \frac{\varepsilon_{s}(\lambda)}{1-\varepsilon_{s}(\lambda)}+\frac{1}{\gamma(\lambda)}\right) \cdot \sigma T_{m}^{4}-S_{m} \cdot \frac{\varepsilon_{s}(\lambda)}{1-\varepsilon_{s}(\lambda)} \cdot J_{m}(\lambda)=\frac{\sigma T_{n}^{4}}{\gamma(\lambda)}
$$

$$
\gamma(\lambda)=\frac{1}{S_{m} \cdot \varepsilon_{s}(\lambda)}+\frac{1}{S_{n}} \cdot\left(\frac{1}{\varepsilon_{n}(\lambda)}-1\right)+\left(\frac{2}{\varepsilon_{s}(\lambda)}-1\right) \cdot \sum_{i=m+1}^{n-1} \frac{1}{S_{i}}
$$

Note that even if the emissivity values now considered may be 171 wavelength dependent, materials still are considered opaque $(\tau=0)$.

Theoretical calculations.-The potential of different thermal 173 shields for radiation heat savings in an industrial Siemens reactor is 174 studied here. The equations presented above are applied to a 36-rod, 175 state-of-the-art Siemens reactor, and as a first approach, the emissivity 176 of the materials is considered constant and wavelength independent. 177 The initial and final diameter of the polysilicon rods is 0.7 and $13 \mathrm{~cm}, \quad 178$ respectively, and their length is $2 \mathrm{~m}$.

In Figure 1 the heat loss due to radiation in Watts (W) throughout a polysilicon deposition process for a constant surface temperature of $1150^{\circ} \mathrm{C}$ is shown; the curves correspond to the case with no thermal shield and four cases with thermal shields. The emissivities of the thermal shields are $0.3,0.45,0.55$ and 0.7 . In Table $\Pi$ the theoretical radiation heat loss savings for the aforementioned thermal shields are presented. The radiation heat loss savings, compared to the heat loss if no thermal shield is considered, are $65.8,52.6,44.3$ and $30.5 \%$ for thermal shield emissivities $(\varepsilon)$ of $0.3,0.45,0.55$ and 0.7 , respectively. This means, assuming that the radiation heat loss is responsible for around $50 \%$ of the total power consumption, that with a thermal shield with $\varepsilon=0.3$ a reduction in power consumption of $32.9 \%$ is obtained, while for $\varepsilon=0.7$ the reduction would be of $15.5 \%$.

The temperature reached by the different thermal shields depending on their emissivity is presented in Figure 2. In all cases, and from the beginning of the process, these temperatures are above $850^{\circ} \mathrm{C}$, which will result in polysilicon deposition on these surfaces. Thus,
150 151 152 153 155 \begin{tabular}{l}
7 \\
8 \\
0 \\
61 \\
\hline
\end{tabular} 162 164 165 166 167 171 172 (1) 6 178 179 180 181 182 183 184 185 186 187 188 189 190 191 192 


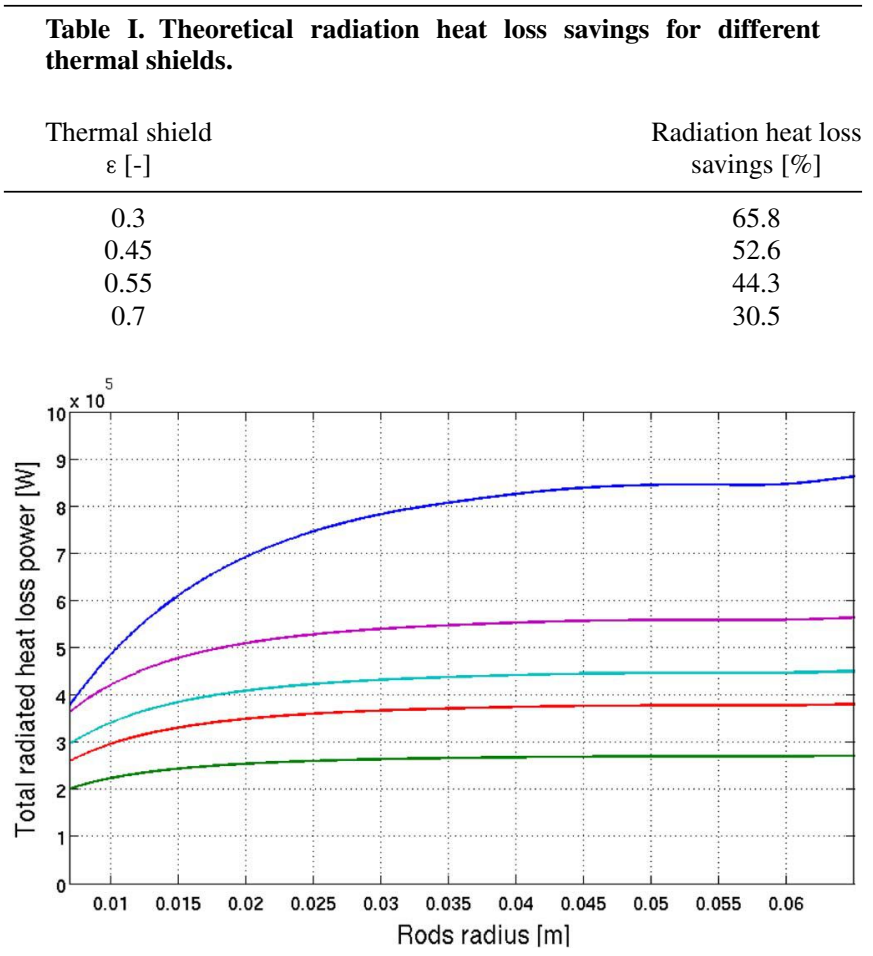

Figure 1. Radiation heat loss for a 36-rod Siemens reactor considering different thermal shields. No thermal shield (blue), $\varepsilon=0.7$ (purple), $\varepsilon=0.55$ (cyan), $\varepsilon=0.45$ (red), $\varepsilon=0.3$ (green).

197 after a few minutes into the deposition process the thermal shield's 198 surface emissivity will be 0.7 , that of silicon at high temperatures. 23 199 Furthermore, contamination issues can arise unless the shields are of 200 a highly pure material. One way to overcome these drawbacks would 201 be to use a thermal shield made of purified silicon $\sqrt{12}$ Not only will it 202 avoid contamination, but one can also collect the silicon deposited on 203 the shields, adding it to the silicon produced in a batch.

The potential of thermal shields can be compared to the use of a 205 polished or a reflective-coated inner wall of a reactor, which will lower 206 the wall emissivity. For a given growth rate, and knowing the power 207 consumption throughout a deposition process, and the initial and the 208 final diameters of the polysilicon rods, the energy consumption in $209 \mathrm{kWh} / \mathrm{kg}$ can be calculated. In Figure 3 the $\mathrm{kWh} / \mathrm{kg}$ ratio for the case 210 of a reflective-coated wall is compared to those considering a silicon 211 thermal shield, no thermal shield and a thermal shield of $\varepsilon=0.3$. For 212 the calculations in Figure 3 the emissivity of the wall and the thermal

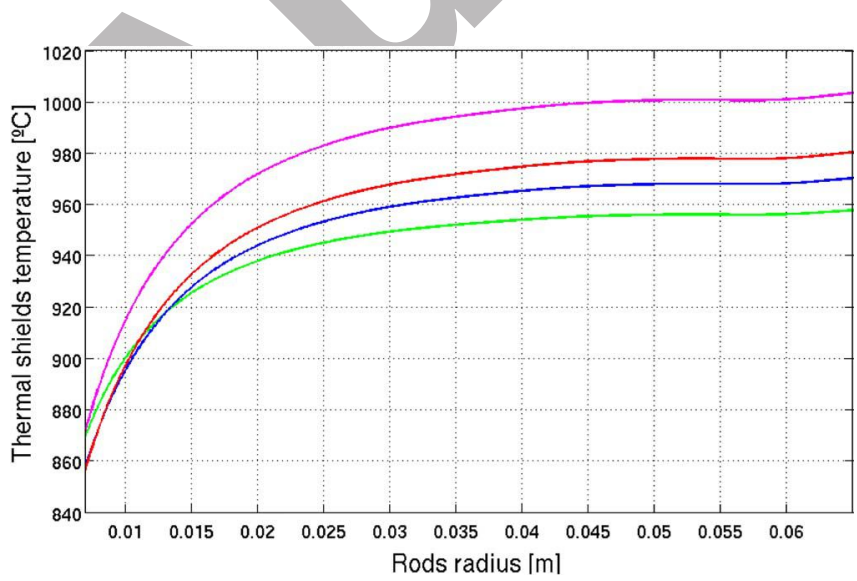

Figure 2. The temperature of thermal shields depending on their emissivity $(\varepsilon)$ throughout a deposition process. Thermal shield emissivities: $\varepsilon=0.7$ (purple), $\varepsilon=0.55$ (red), $\varepsilon=0.45$ (blue), $\varepsilon=0.3$ (green).

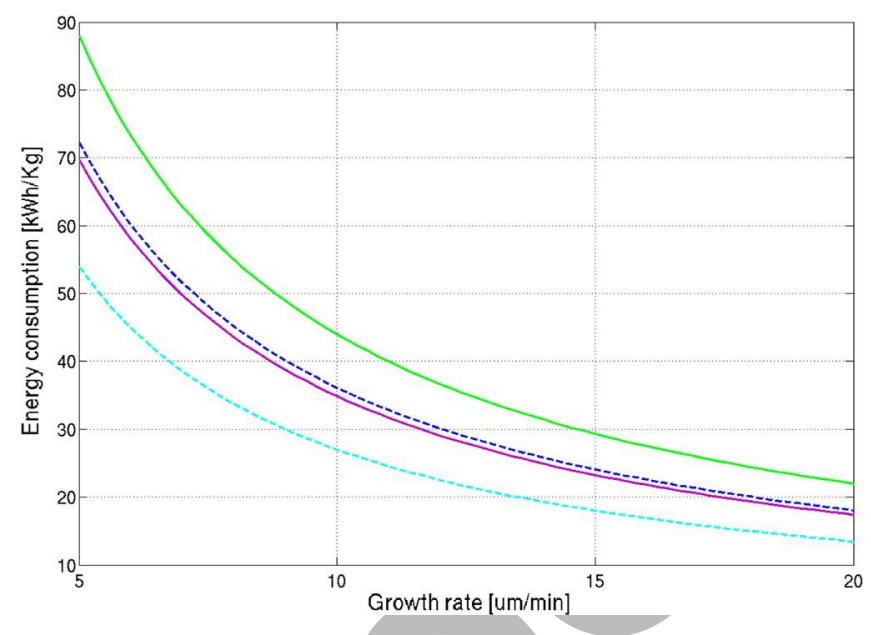

Figure 3. Total power consumption of a 36-rod Siemens reactor for different growth rates considering: no thermal shield $-\varepsilon_{\text {wall }}=0.5$ - (green), silicon thermal shield $-\varepsilon=0.7$ - (purple), thermal shield with $\varepsilon=0.3$ (cyan) and no thermal shield and polished reactor wall $-\varepsilon_{\text {wall }}=0.3$ - (blue).

shields is considered constant throughout a deposition process; and the 213 radiation heat loss is $50 \%$ of the total power consumption. The lowest 214 $\mathrm{kWh} / \mathrm{kg}$ ratio is obtained for a low emissivity thermal shield, and the 215 $\mathrm{kWh} / \mathrm{kg}$ ratio for that with a silicon thermal shield and a polished 216 inner wall are quite close. However, note that the low emissivity 217 thermal shield and the polished walls will not maintain their initial 218 emissivities for more than a short period of time, as silicon or a silane- 219 based compound will deposit. After a few minutes into the deposition 220 process the blue curve will start to move slowly upwards until it 221 reaches the green curve; and the cyan curve will quickly move to 222 behave like the purple curve. Thus, the effect of a thermal shield is 223 more efficient in terms of energy savings than considering a polished 224 reactor wall; this statement is true even when considering a high initial 225 emissivity value for the thermal shield (e.g., $\varepsilon=0.7$ ).

\section{Laboratory Scale Experiments}

227

A number of experiments considering thermal shields are con- 228 ducted in a laboratory Siemens reactor,, and the effect on radiation 229 heat savings obtained is put forward.

Since the temperature of the thermal shield in the laboratory reac- 23 tor will be lower than in the industrial case, the laboratory prototype 232 allows us to test the effect of thermal shields with different emissivi- 233 ties. The key parameter for the selection of the thermal shield material 234 is the emissivity $(\varepsilon)$; but also, the material selected must be easily 235 machinable, and available with the geometries and thickness required 236 for its assembly inside the reactor chamber, so its mechanical strength 237 must be assured. The following materials are evaluated: molybdenum, 238 boron nitride, stainless steel, aluminum oxide (alumina), zirconium, 239 graphite foil and silicon. Some of the relevant properties of these 240 materials are presented in Table $\overline{\mathrm{II}}$; the values shown are considered ${ }_{24}$ wavelength independent since this dependence is unknown.$$
242
$$

Table II. Properties of different materials considered for the thermal shields. $\underline{25,26}$

\begin{tabular}{lccc} 
Material & $\begin{array}{c}\varepsilon[-] \\
\left(\mathrm{T}=25^{\circ} \mathrm{C}\right)\end{array}$ & $\begin{array}{c}\varepsilon[-] \\
\left(\mathrm{T} \sim 600^{\circ} \mathrm{C}\right)\end{array}$ & $\begin{array}{c}\text { Ease of } \\
\text { machining }\end{array}$ \\
\hline Molybdenum & - & $0.8-0.9$ & Medium \\
Stainless steel & $0.6-0.8$ & $0.7-0.9$ & Low \\
Alumina & - & $0.3-0.4$ & Medium \\
Boron nitride & $0.9-0.95$ & - & Medium \\
Zirconium & - & $0.1-0.3$ & High \\
Graphite foil & $0.7-0.9$ & $0.4-0.6$ & Low \\
Silicon & - & 0.7 & Medium
\end{tabular}




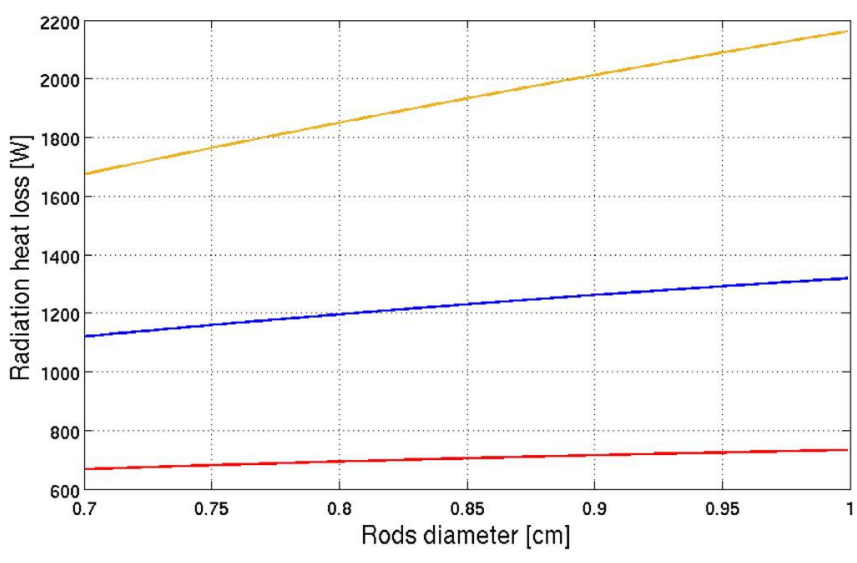

Figure 4. Radiation heat loss in the laboratory Siemens reactor for a 7-rod configuration considering a silicon thermal shield (blue), a low emissivity thermal shield $-\varepsilon=0.3$ - (red) and without thermal shield (orange).

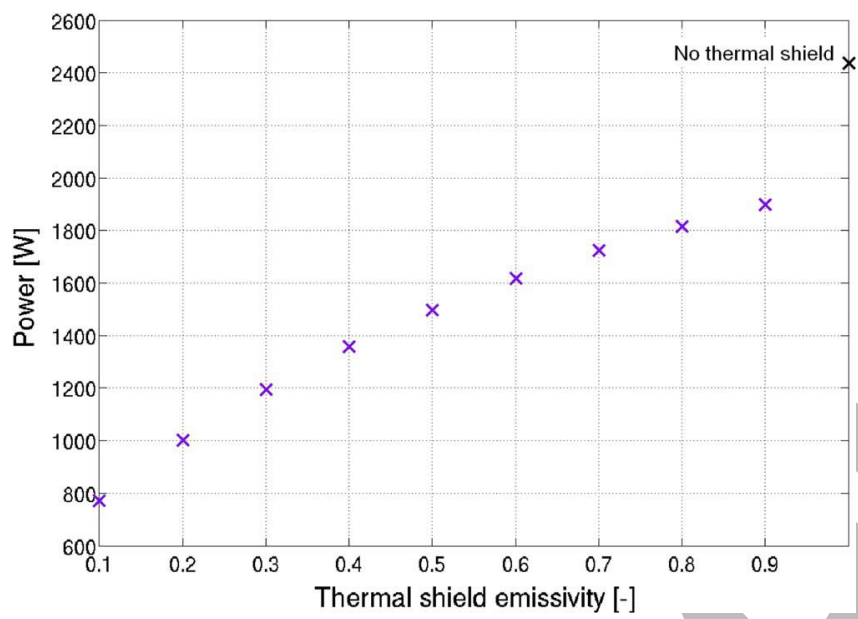

Figure 5. Laboratory Siemens reactor power consumption $(\mathrm{P})$ predicted in theory for different thermal shield emissivities and for the case of no thermal shield considering a 7-rod configuration.

First, the radiation heat loss equations with thermal shields are applied to the laboratory Siemens reactor. The radiation heat loss 245 for a 7-rod configuration with a low emissivity shield, with a silicon 246 thermal shield and without thermal shield is presented in Figure 4: it 247 can be seen how the lowest radiation heat losses are obtained for a low 248 emissivity thermal shield. The temperatures reached by the thermal 249 shields are in the range of $600-750^{\circ} \mathrm{C}$.

250 The power consumption predicted by the model for different ther251 mal shield emissivities and for that of no thermal shield, are presented 252 in Figure 5. For these calculations a constant deposition temperature 253 of $1100^{\circ} \mathrm{C}$, the same growth rate and the same duration of the depo254 sition processes, is considered, thus averaging the measured data. It 255 can be seen that the lower emissivity of the thermal shield, the higher 256 radiation heat savings.

Experiments with thermal shields.-A 7-rod configuration is cho258 sen as a compromise solution between a dense compactness - a large 259 number of rods - and the size of the reactor chamber. The length of 260 the rods is $10 \mathrm{~cm}$ and their initial diameter is around $0.7 \mathrm{~cm}$.

From the thermal shield materials listed in Table III, the following 62 have been selected for testing: silicon, alumina and stainless steel. 263 Different thickness of the selected materials are considered, and in 264 some cases the outer surface of the thermal shields is silver coated

${ }^{\mathrm{b}}$ The silver coatings deposited are a few hundred nanometers thick.

\begin{tabular}{|c|c|}
\hline Experiment name & Description \\
\hline No shield (No) & Without any thermal shield \\
\hline Silicon shield (Si1) & $\begin{array}{l}\text { Multi-crystalline silicon thermal } \\
\text { shield ( } 290 \mu \mathrm{m} / \text { layer; } 3 \text { layers })\end{array}$ \\
\hline Silicon shield (Si2) & $\begin{array}{l}\text { Mono + Multi-crystalline silicon thermal } \\
\text { shield }(400+290 \mu \mathrm{m} ; 1+1 \text { layers })\end{array}$ \\
\hline Silicon shield (Si3) & $\begin{array}{l}\text { Mono + Multi-crystalline silicon thermal } \\
\text { shield }(400+290 \times 4 \mu \mathrm{m} ; 1+4 \text { layers })\end{array}$ \\
\hline Alumina shield (Alu1) & $\begin{array}{l}\text { Alumina shield } \\
\text { (1 mm thick) }\end{array}$ \\
\hline Alumina shield (Alu2) & $\begin{array}{l}\text { Alumina shield silver coated } \\
\text { (1 mm thick) }\end{array}$ \\
\hline Steel shield (Ste) & $\begin{array}{l}\text { Stainless steel shield } \\
\text { (1 mm thick) }\end{array}$ \\
\hline
\end{tabular}

\begin{tabular}{|c|c|c|c|c|}
\hline Experiment & (No) & (Si1) & $(\mathrm{Si} 2)$ & $(\mathrm{Si} 3)$ \\
\hline $\mathrm{T}_{\text {deposition }}\left[{ }^{\circ} \mathrm{C}\right]$ & 1106 & 1101 & 1108 & 1108 \\
\hline Si deposited [gr] & 50.7 & 61.9 & 59.3 & 59.8 \\
\hline Power $_{\text {average }}$ & 2343 & 1979 & 2042 & 2122 \\
\hline Time [min] & 392 & 406 & 385 & 375 \\
\hline $\mathrm{T}_{\text {wall }}\left[{ }^{\circ} \mathrm{C}\right]$ & 280 & 233 & 184 & 181 \\
\hline $\mathrm{T}_{\text {shield }}\left[{ }^{\circ} \mathrm{C}\right]$ & - & 678 & 641 & 616 \\
\hline Growth rate $[\mu \mathrm{m} / \mathrm{min}]$ & 2.9 & 3.5 & 3.6 & 3.6 \\
\hline Consumption $[\mathrm{kWh} / \mathrm{kg}]$ & 311 & 216 & 221 & 222 \\
\hline Energy savings [\%] & - & 28.4 & 26.8 & 26.5 \\
\hline
\end{tabular}

The emissivity of silver is very low $(\varepsilon \sim 0.02-0.05)$, so if this coating 265 withstands the process temperatures, it will act as a mirror making a 266 non-opaque body behave almost as if it were.

The relevant data related to these experiments is presented in the 268 following tables. First, the different thermal shields are described and 269 related to their corresponding label in Table III. Then, the experimental 270 results are grouped together in 'silicon shields' and 'alumina and 271 stainless steel shields'; Tables IV] and V] respectively.

From the data presented in Table IV, the energy savings obtained 273 with the different silicon thermal shields are similar. The reduction 274 in the $\mathrm{kWh} / \mathrm{kg}$ ratio obtained considering thermal shields related to 275 experiment (No) are between 26.5 and $28.4 \%$. All these experiments 276 were conducted under similar conditions and their duration is similar. 277 Despite the fact that the deposition surface temperature is in all cases 278 around $1100^{\circ} \mathrm{C}$, there is a difference in the growth rate obtained in 279 experiment (No). This is so because the presence of a thermal shield 280 changes the distribution of the gas temperature, and higher tempera- 281 tures are achieved in the gas surrounding the silicon rods.

From the data presented in Table V, the energy savings in $\mathrm{kWh} / \mathrm{kg}, \quad 283$ compared with experiment (No), vary between 15.1 and $30.7 \%$. The 284

\begin{tabular}{|c|c|c|c|c|}
\hline Experiment & (No) & (Alu1) & (Alu2) & (Ste) \\
\hline $\mathrm{T}_{\text {deposition }}\left[{ }^{\circ} \mathrm{C}\right]$ & 1106 & 1107 & 1108 & 1098 \\
\hline Si deposited [gr] & 50.7 & 65.3 & 53.7 & 49.3 \\
\hline Power $_{\text {average }}$ & 2343 & 2333 & 1669 & 1915 \\
\hline Time [min] & 392 & 430 & 404 & 388 \\
\hline $\mathrm{T}_{\text {wall }}\left[{ }^{\circ} \mathrm{C}\right]$ & 280 & 142 & 175 & 152 \\
\hline $\mathrm{T}_{\text {shield }}\left[{ }^{\circ} \mathrm{C}\right]$ & - & 736 & 705 & 570 \\
\hline Growth rate $[\mu \mathrm{m} / \mathrm{min}]$ & 2.9 & 3.6 & 3.2 & 3.0 \\
\hline Consumption $[\mathrm{kWh} / \mathrm{kg}]$ & 311 & 256 & 205 & 251 \\
\hline Energy savings [\%] & - & 15.1 & 30.7 & 16.8 \\
\hline
\end{tabular}


$285 \mathrm{kWh} / \mathrm{kg}$ values in the laboratory scale reactor are several times higher 286 than those found in industrial processes, mainly since the process pres-

289 from that expected from its theoretical $\varepsilon$ (further explanations will be 290 presented in Discussion on energy savings section).

291 Lastly, in experiments conducted with silicon thermal shields etch292 ing is detected on the surface of the shields. This is attributed to the 293 presence of $\mathrm{SiCl}_{4}$ as a by-product of the reduction reaction. The oc294 currence of this phenomenon versus polysilicon deposition depends 295 on the mol fraction of $\mathrm{SiCl}_{4}$, which will depend on the deposition sur296 face temperature ${ }^{[27,28} \mathrm{High} \mathrm{SiCl}_{4}$ concentrations and low temperatures 297 favor the etching. However, as already explained, under industrial de298 position conditions the temperature of the thermal shields will be 299 such that polysilicon will be deposited on the thermal shields, and no 300 etching is expected.

$301 \quad$ Discussion on energy savings.-From the above, energy savings 302 have been confirmed for the 7-rod configuration experiments consid303 ering different thermal shields.

304 If the experimental data from Tables IV and $\mathrm{V}$ (average power 305 consumption and energy savings) is compared with the theoretical cal306 culations for different thermal shields (Figure 5), a good agreement 307 for the case of no thermal shields is obtained; differences between 308 both values are under $3.8 \%$. Note that our calculations consider con309 stant deposition conditions, while the experimental conditions of the 310 deposition process vary slightly from one experiment to another. $3132333 \mathrm{~W}$ and $15.1-30.7 \%$, respectively. According to data presented in 314 Figure 5, the previous values correspond to thermal shield emissivities 315 above 0.6 . In the case of the silicon thermal shields, the energy savings 316 obtained correspond to $\varepsilon=0.7-0.8$, for the alumina shields to $\varepsilon>$ 3170.9 , for the silver coated alumina shield to $\varepsilon=0.6-0.7$; and for the 318 stainless steel shield to $\varepsilon>0.9$. These $\varepsilon$ values do not correspond 319 to those found in the bibliography assuming the gray body approach, 320 which is no surprise since the gray body approach simplifies much of 321 the radiative behavior of real bodies.

Reflectance, transmittance and emissivity measurements.-With the aim of clarifying the real emissivity of the thermal shield materials tested in the laboratory Siemens reactor, reflectance $(\rho)$ and transmittance $(\tau)$ measurements for different $\lambda$ are taken. Both, $\rho(\lambda)$ and $\tau(\lambda)$, can be measured directionally or integrated; in the present case integrated measurements are suitable since the materials considered do not have specular surfaces. These measurements are conducted at room temperature.

In Figure 6 the integrated transmittance measurements, within the wavelength range $\lambda \in(2.5-20) \mu \mathrm{m}$, for different thermal shields are presented. In all cases, noticeably for the silicon shield, the integrated transmittance is $\tau \neq 0$. Measurements for a silicon, alumina and stainless steel thermal shields are presented in Figure 6. The integrated transmittance measured is on average $41.3,8.1$ and $0.5 \%$ for the

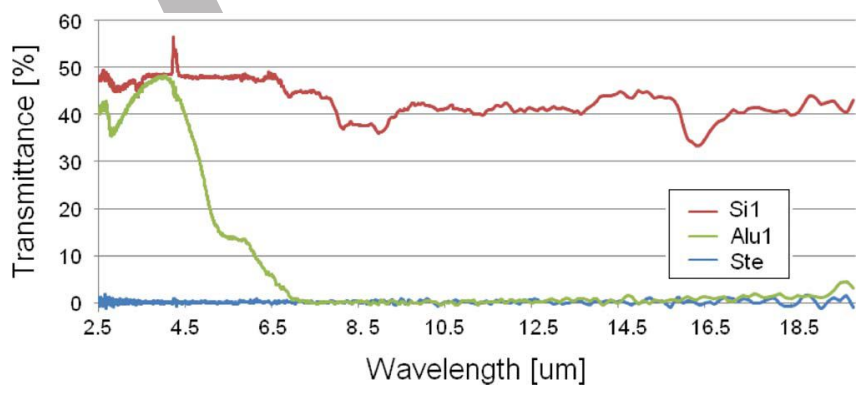

Figure 6. Integrated transmittance $(\tau)$ of: $290 \mu \mathrm{m}$ multi-crystalline silicon (red), $1 \mathrm{~mm}$ alumina (green) and $1 \mathrm{~mm}$ stainless steel (cyan).

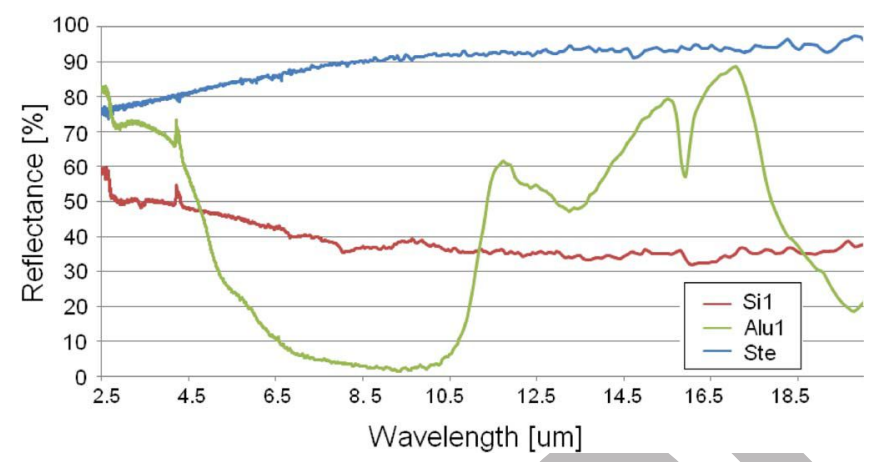

Figure 7. Integrated reflectance ( $\rho$ ) of: $290 \mu \mathrm{m}$ multi-crystalline silicon (red), $1 \mathrm{~mm}$ alumina (green) and $1 \mathrm{~mm}$ stainless steel (cyan).

$290 \mu \mathrm{m}$ multi-crystalline, $1 \mathrm{~mm}$ alumina and $1 \mathrm{~mm}$ stainless steel 336 samples, respectively.

Integrated reflectance measurements are also conducted; $\rho(\lambda)$ for $\lambda \in(2.5-20) \mu \mathrm{m}$ for silicon, alumina and stainless steel are presented 339 in Figure 7. From Figure 7, the averaged reflectance of the silicon 340 sample is $40 \%$, while the respective values for that of alumina and the 341 stainless steel samples are 48.1 and $92.8 \%$, respectively.

From the average values of the aforementioned transmittance and 343 reflectance integrated measurements, only the stainless steel sample 344 presents a very low transmittance. Materials experimentally tested in 345 the laboratory Siemens reactor at room temperature definitely do not 346 behave as gray bodies, and similar behavior can be expected at higher ${ }_{347}$ temperatures. $\frac{18,199}{1}$ The latter explains the differences between the pre- ${ }_{348}$ dicted energy savings and the empirically obtained ones. The next 349 section discusses the effect that the wavelength-dependent emissivi- 350 ties can have on the radiation heat losses.

\section{5}

\section{Discussion on the Contribution to the Radiation Heat Loss Model 352}

The model for radiation heat loss is applied here for the radi- 353 ation heat loss calculations of a 36-rod industrial Siemens reactor, 354 considering thermal shields that do not behave as gray bodies. Two 355 hypothetical thermal shields with an averaged $\varepsilon(\lambda)=0.7$ are con- 356 sidered, with an emissivity variation presented in Figure 8, It can be 357 seen that $\varepsilon(\lambda)$ of material 1 is approximately constant, while $\varepsilon(\lambda)$ of 358 material 2 is heavily dependent on the wavelength.

The radiation heat loss for $\lambda \in(0.1,20) \mu \mathrm{m}$, calculated for a 36-rod 360 industrial Siemens reactor, is presented in Figure 9. The two scenarios 361 presented; hereinafter scenarios 1 and 2, correspond to material 1 and 362 material 2 thermal shields, respectively. In both cases, the radiation 363

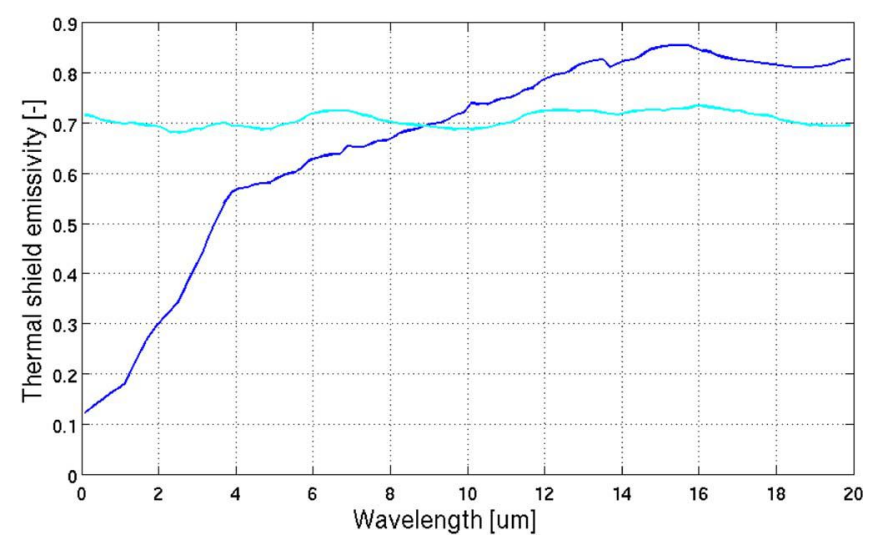

Figure 8. Emissivity $\varepsilon(\lambda)$ for two different thermal shield materials: material 1 (cyan) and material 2 (blue). In both cases, the averaged $\varepsilon(\lambda)=0.7$. 

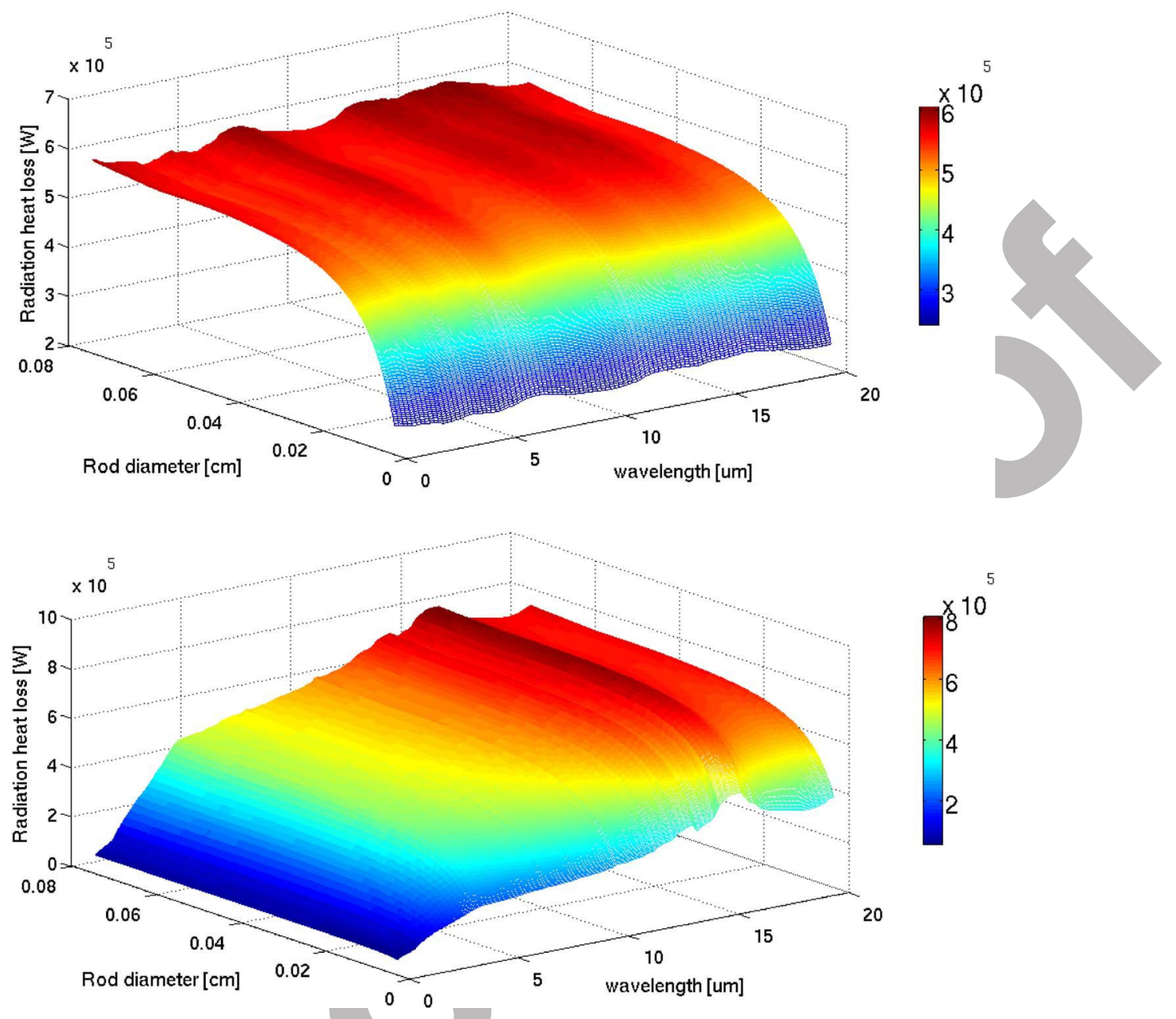

Figure 9. Radiation heat loss for different wavelengths for two different thermal shield materials: material 1 (top) and material 2 (bottom).

364 heat loss variation with $\lambda$ is similar to the corresponding variation in $365 \varepsilon(\lambda)$ of the shield material considered.

When the surfaces presented in Figure 9 are integrated along the entire radiation spectrum, the radiation heat loss values presented in Figure 10 for scenarios 1 and 2 are obtained. This curves are pre-

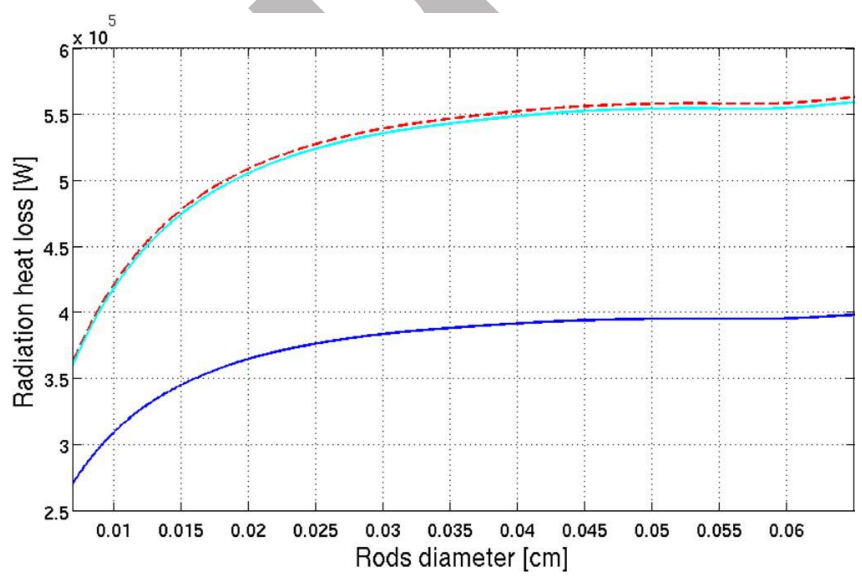

Figure 10. Radiation heat loss for different thermal shield materials: material 1 (cyan), material 2 (blue), obtained by integrating Figure 9 along all the radiation spectrum. The case of a material with a constant $\varepsilon(\lambda)=0.7$ is presented for comparison (red). sented together with the corresponding curve if a constant emissivity 369 for the thermal shield $\varepsilon(\lambda)=0.7$ is considered - scenario 3. It can 370 be appreciated how the scenarios 1 and 3 are quite close, but great 371 differences in radiation heat loss are obtained between scenarios 1 and 372 3 , and scenario 2 ; the averaged differences are above $25 \%$. As regards 373 the thermal shield temperature, results obtained for scenarios 1 and $3 \quad 374$ are also quite close; the temperature of the shields is around $870^{\circ} \mathrm{C} \quad 375$ at the beginning of the deposition process, increasing rapidly until it 376 reaches around $1000^{\circ} \mathrm{C}$ at the end of the process. In scenario 2, the 377 thermal shield temperature has a similar behavior with temperature 378 values that go from 860 to $975^{\circ} \mathrm{C}$.

The aforementioned differences above are explained since not all wavelengths contribute to the same extent to the radiation heat loss; 381 in particular for these three scenarios, the greatest contribution of $\varepsilon(\lambda)$ occurs in the range $\lambda \in(1-6) \mu \mathrm{m}$.

These results highlight the importance of having reliable data on 384 the emissivities in the relevant range of wavelengths, and for the 385 application of silicon CVD, at deposition process temperatures, which 386 remains pending.

\section{Conclusion}

A radiation model for heat loss calculations in a Siemens-type reactor has been presented, in which the fraction of energy leaving a certain surface that arrives at another surface is evaluated using the geometric Hottel crossed-string method, and the effect of the emissivity variation with the wavelength is taken into account. A significant

0 1

372

373

374
375
376
377 
394 potential for reducing radiation heat loss in Siemens reactors has been 395 identified, considering different thermal shields. The model shows 396 that materials with lower surface emissivities lead to higher radiation 397 heat loss savings. The effect of a thermal shield is also more efficient 398 in terms of energy savings than considering a polished reactor wall, 399 even for a thermal shield with a high initial emissivity value.

$400 \quad$ Experiments considering different thermal shields are conducted 401 in a laboratory Siemens reactor. It has been experimentally shown 402 that significant energy savings in the polysilicon deposition process 403 are obtained.

404 Silicon thermal shields have some advantages in terms of pre405 venting contamination and collecting the silicon deposited on them, 406 and energy savings of between $26.5-28.5 \%$ have been experimentally 407 proven.

408 Reflectance and transmittance measurements as a function of 409 wavelength are taken for the materials tested, proving that they do 410 not behave as gray bodies at room temperature, and similar behavior 411 can be expected at higher temperatures. Results highlight the impor412 tance of having reliable emissivity data on the materials involved at 413 deposition temperatures, which remains pending.

415 The Spanish Ministerio de Economía y Competitividad is acknowl416 edged for its support through the IPT 2012-0340-120000 project and 417 Comunidad de Madrid through the MADRID-PV S2013/MAE-2780 418 project.

419 Ángel Morales, from the Centro de Investigaciones Energéticas 420 Medioambientales y Tecnológicas (CIEMAT), Spain is acknowledged 421 for his contribution to the reflectance and transmittance measurements.

\section{References}

42

1. K. Hesse, E. Schindlbeck, E. Dornberger, and M. Fischer, "Status and Development of Solar-Grade Silicon Feedstock," 24th European Photovoltaic Solar Energy Conference, 883 (2009).

2. R. Berstein, "A shortage hits solar power," The Wall Street Journal, (2006)

3. A. F. B. Braga, S. P. Moreira, P. R. Zampieri, J. M. G. Bacchin, and P. R. Mei, "New processes for the silicon production of solar-grade polycrystalline silicon: A review," Solar Energy Materials and Solar Cells, 92, 418 (2008).

4. C. Wang, T. Wang, P. Li, and Z. Wang, "Recycling of $\mathrm{SiCl}_{4}$ in the manufacture of granular polysilicon in a fluidized bed reactor," Chemical Engineering Journal, 220, (2013).
5. B. Ceccaroli and O. Lohne, Handbook of Photovoltaic Science and Engineering, in: 432 Solar Grade Silicon Feedstock, 2nd Edition, pp. 169-217 (2011).

6. E. Alsema and M. de Wild-Scholten, "Reduction of the environmental impacts in 434 crystalline silicon module manufacturing," 22nd European Photovoltaic Solar Energy Conference and Exhibition, pp. 829-836, (2007).

7. S. Singer, "Photovoltaics: Getting Cheaper," EcoQueen of Green, (2007). the gigawatt era," Journal of Crystal Growth, 393, 2 (2014).

9. A. Ramos, A. Rodríguez, C. del Cañizo, J. Valdehita, J. C. Zamorano, and A. Luque, "Heat losses in a CVD reactor for polysilicon production: Comprehensive model and experimental validation," Journal of Crystal Growth, 402, 138 (2014).

10. H. Griss, B. Caussat, H. Vergnes, and J. P. Couder, "An improvement in the behavior of LPCVD reactors: the dead zones reducers," Chemical Vapor Deposition: Proceedings of the Fourteenth International and EUROCVD-11, 194-201, 1997.

11. M. A. Gallivan, D. G. Goodwin, and R. M. Murray, "A Design Study for Thermal Control of a CVD Reactor for YBCO," Proceedings of the 1998 IEEE International Conference on Control Applications, Italy, 1998.

12. C. del Cañizo, G. del Coso, A. Luque, and J. C. Zamorano, "Thermal shield for silicon production reactors," [Pat.: WO 2012025513 A1], (2012).

13. G. Pazzaglia, M. Fumagalli, and M. Kulkarni, "Bell jar for siemens reactor including thermal radiation shield," [Pat.: WO 2011128729 A1], (2011).

14. J. Lee, J. Kim, S. Lee, and L. C. Winterton, "Chemical vapor deposition reactor having a radiant heat barrier layer for improving energy efficiency," [Pat.: WO 2011068283 A1], (2011).

15. F. Kreith and M. Bohn, Heat transfer by radiation, Principles of heat transfer, 6th Ed., Thomson, pp. 539-626, (2002).

16. M. F. Modest, Radiative heat transfer, 2nd Ed., Academic Press, 2003.

17. D. K. Schroder, Semiconductor Material and Device Characterization, 3rd Ed., Wiley-IEEE Press, 2006.

18. T. Paloposki and L. Liedquist, Steel emissivity at high temperatures, VTT Tiedotteita - Research Notes 2299, pp. 81, 2005.

19. C. E. Kennedy, Review of Mid- to High-Temperature Solar Selective Absorber Materials. National Renewable Energy Laboratory (NREL), 2002.

20. G. del Coso, C. Cañizo, and A. Luque, "Radiative energy loss in a polysilicon CVD reactor," Solar Energy Materials \& Solar Cells, 95, 1042 (2011).

21. A. Ramos, C. del Cañizo, J. Valdehita, J. C. Zamorano, and A. Luque, "Radiation heat savings in polysilicon production: Validation of results through a CVD laboratory prototype,"Journal of Crystal Growth, 374, 5 (2013).

22. R. Siegel and J. R. Howell, Thermal radiation heat transfer, McGraw-Hill, Siegel72, 1972.

23. G. del Coso, "Chemical decomposition of silanes for the production of solar grade silicon," Ph.D. Thesis, Universidad Politécnica de Madrid - ETSI Telecomunicación, 2010.

24. A. Ramos, C. del Cañizo, J. Valdehita, J. C. Zamorano, A. Rodríguez, and A. Luque, "Exploring polysilicon deposition conditions through a laboratory CVD prototype," Physica Status Solidi C, 10 (2012)

25. D. R. Lide (Ed.), CRC Handbook of Chemistry and Physics, National Institute of Standards and Technology, 2005.

26. G. G. Gubareff, J. E. Janssen, and R. H. Torborg, Thermal Radiation Properties Survey, Honeywell Research Center, 1960

27. S. Grove, Physics and Technology of Semiconductor Devices, Willey, 1967.

28. S. M. Sze, Semiconductor Devices: Physics and Technology, 2nd Ed., Willey, 2001. 437 439 442 444 446
447 448 449
450 451 454 455 455
456 457 458 458 459
460 461 463 464 465 466 467 468 469 470 471 471 472
473 474 475 475
476 477 